\title{
Observation of microstructures of atmospheric ice using a new replica technique
}

\author{
ML.A. Pervier ${ }^{\mathrm{a}}$, H. Pervier ${ }^{\mathrm{a}}$, D.W. Hammond ${ }^{\mathrm{a}}$, \\ ${ }^{a}$ Cranfield University, Cranfield,MK43 OAL,UK
}

\begin{abstract}
A replication technique has been developped to study the microstructure of atmospheric ice based on the use of nail varnish rather than more harmful materials. The potential of the technique was demonstrated by obtaining and reporting microstructures for impact ice grown on metal surfaces in an icing tunnel under a range of cloud conditions. The technique reveals grain structure, growth striations, porosity and etch features which may indicate an aspect of crystalographic orientations.
\end{abstract}

Keywords:

atmospheric ice, impact ice, microstructure, grain size

In order to study the mechanical response of atmo- ${ }_{27}$ spheric ice including adhesion to a substrate, knowledge 28 of the ice's microstructure is of prime importance. At- 29 mospheric ice by its nature is very different from other 30 types of ice and varies significantly in its microstruc- 31 ture. Impact ice is formed by the freezing of super- 32 cooled droplets when they strike a surface. Lake ice, sea ice or other types of ice grown by cooling a body 34 of water are dominated by the fact that the cooling is 35 limited by thermal conduction effects. This generally 36 results in producing much coarser grain size, modifying ${ }_{37}$ grain aspect ratio and influencing pore size and distribu- 38 tion compared to impact ice.

\section{Previous work}

While knowledge of the microstructure of ice is important for understanding the fracture process and ice adhesion, as it is in many structural materials, only a small number of authors have reported such data. This is perhaps due to the difficulty of handling a material which is easily broken and modified by a slight change of temperature.

Different methods to observe ice microstructure have been reported by Blackford (2007). Depending on the methods used, different level of details can be studied and they all present their advantages and drawbacks. The most direct method, and most commonly used, is

URL: m.pervier@cranfield.ac.uk (ML.A. Pervier), d.w.hammond@cranfield.ac.uk (D.W. Hammond)

Preprint submitted to Cold Region Science and Technology

to make and observe a thin slice of ice under a microscope using reflecting or transmitted light. With a resolution of $1 \mu \mathrm{m}$, this method allow the observation of the size, shape and distribution of the grains as well as the location and distribution of the impurities. The use of polarized light gives more information on the crystallographic orientation of each grains (Durand, 2006). The main drawbacks of the methods resides in the preparation of thin sections of ice and in avoiding any transformation of the ice microstructure with time.

To overcome the difficulties of handling and keeping the sample intact, several authors (Nasello et al., 1987; List et al., 1970; Higuchi, 1958; Fortin and Perron, 2009) have used a replication technique based on a solution of formvar disolved in a solvent such a chloroform. This is known to be toxic and carcinogenic (Takahashi and Fukuta, 1987). Other authors (Takahashi and Fukuta, 1987; Kriston et al., 2016) have used an alternative to formvar. Takahashi and Fukuta have successfully replicate ice crystal using common plastics dissolved in solvent and discussed the ability of each combination. Best results were obtained using plexiglas with ethylene dichloride or trichloroethylene and polystyrene with 1,1,1-trichloroethane or carbon tetrachloride. While these solvants have the advantage of remaining clear, compared to formvar, due to the absence of water soluble ingredients, they are still harmfull. Kriston et al. reported work on the effect of the friction of rubber tyre on ice. They replicated the ice surface by using a dental casting material called vinyl polysiloxane. They obtained a negative replica of the 

alloy A12024-T3 (figure 1). The substrate surfaces were

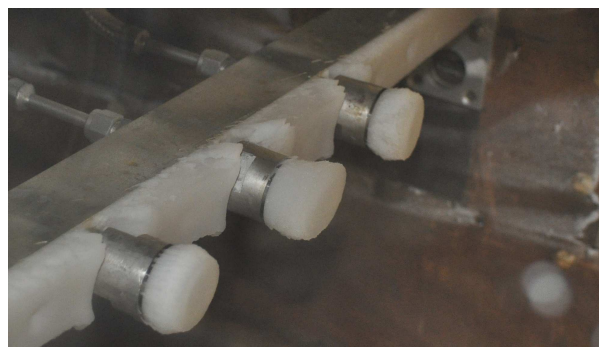

Figure 1: Samples of ice accreted on the front face of metallical cylinders $\left(\mathrm{T}=-15^{\circ} \mathrm{C}, \mathrm{V}=80 \mathrm{~m} \cdot \mathrm{s}^{-1}, \mathrm{LWC}=0.3 \mathrm{~g} \cdot \mathrm{m}^{-3}, \mathrm{MVD}=20 \mu \mathrm{m}\right)$

ice surface which was then used as a mould to obtain 95 a positive replica using polyurethane. Depending on 96 the level of detail required, optical microscope, SEM 97 or white light interferometer was used. Grain and sub- 98 grain boundaries, etch pits, frost and scratches due to the effect of multiple sweeping of rubber on ice were observed.

In the present paper, a similar technique has been tested but by replacing the solution of formvar with less harmfull nail varnish. While nail varnish composition generally includes some kind of alcohol and acetone, the proportion of these in some nail varnish is low enough to not cause any damage to the ice surface. A description of the method and the level of detail that can be obtained is presented in the following.

\section{Methodology}

Samples of ice were produced in Cranfield Icing Tunnel (Hammond, 2003) as a by product of mechanical tests. Ice was accreted on the front face of cylinders 104 made of either Titanium alloy Ti-6Al-4V or Aluminium 105

polished to mirror finish with a resulting roughness of $0.3 \mu \mathrm{m}(\mathrm{Ra})$. The pieces of ice were detached from the metallic surface using a hammer and a chisel and positioned at the bottom of a chest freezer straight after removal. The removal was completely adhesive meaning that the substrate surface was visually free of ice.

The ice pieces were kept in loose fitting plastic bags in the freezer at a temperature of $-18^{\circ} \mathrm{C}$ for a couple of weeks before the surface were replicated using the nail varnish technique. During this resting time, sublimation will have occured revealling the grain boundaries as this process is faster along the grain boundaries than within the body of the grain. A layer of nail varnish was applied to the surface of interest using the small brush provided with the nail varnish bottle. After a drying time of 14 to 20 hours, the nail varnish layer was peeled off the ice giving the replica including imprint of the peaks and groves present on the ice surface. Three different types of replicae were collected representing different orientations and regions of each ice specimen. These are shown in figure 2 .
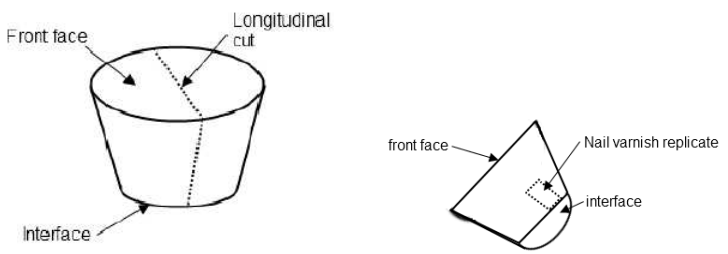

Figure 2: surface replica of the ice sample

The three types of replicas collected are referred as follows:

- the surface which was in contact with the substrate (called the interface)

- the surface at the other extremity (called the front surface)

- a longitudinal surface which was obtain by cutting the ice piece normal to the substrate surface

In most of the samples, the interface and the front surface were both replicated directly without the need to prepare the sample further. When the surface was not flat enough (it is mostly the case with the front surface of glaze ice), the ice surface was manually polished using carbide paper. As this process leaves some groves onto the surface, the ice piece needed to be left in the freezer for a further week to allow the sublimation process to remove them. The longitudinal surface was cut in a cooled environment $\left(-15\right.$ to $\left.-20^{\circ} \mathrm{C}\right)$ using a saw with relatively large teeth to help the sawing action. The saw was also cooled prior to any sample cutting. One of the two halves was chosen and further ground with fine grain metalurgical grade silicon carbide paper (180 grit). The sample was then left to sublimate for several days in the freezer. The nail varnish was applied using from the interface side to the front surface (figure 2).

Replicas were prepared for the microscopic observation by sandwiching them between a microscope slide and a cover slip. They were observed under transmited unpolarised light in a microscope and photos taken. The photos were stitched together using the software iMerge ${ }^{1}$. The average grain size was obtained, for each case, by

\footnotetext{
${ }^{1}$ iMerge is an images stacking and mosaic-making software conceived primarily for astronomical purpose
} 


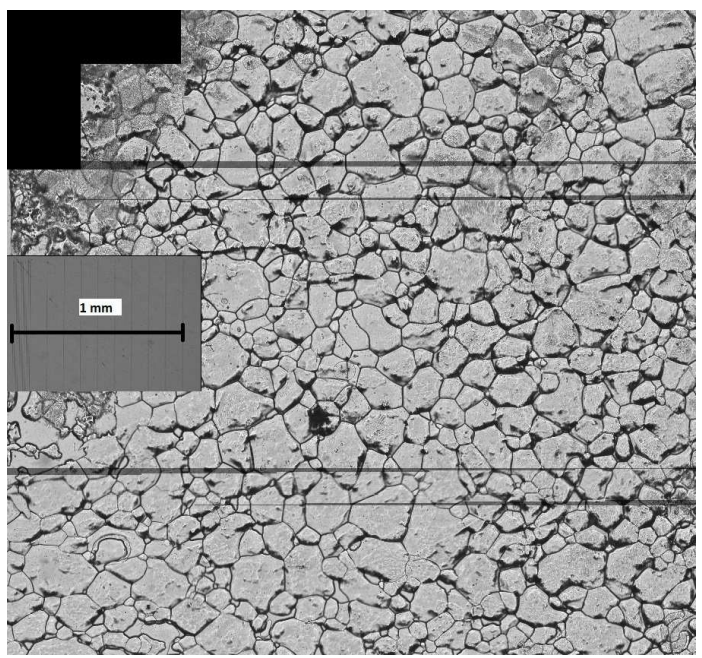

Figure 3: Microstructure of the interface of ice built on a mirror polished surface finish titanium alloy substrate $\left(T=-5^{\circ} \mathrm{C}, \mathrm{LWC}=0.3\right.$ $\mathrm{g} . \mathrm{m}^{-3}, V=50 \mathrm{~m} \cdot \mathrm{s}^{-1}, M V D=20 \mu \mathrm{m}$ )

using the mean linear intercept method on ten separate measurements (standard deviation of 10\%).

\section{Description of the microstructure obtained}

The images collected allow us to see the grain boundaries of the ice sample as well as the presence of air pockets. In some cases, when the grains dimensions are large, some thermal etch pits becomes visible.

\subsection{Different surfaces of the same piece of ice}

Figure 3 to 5 present the three different surfaces of interest of the same piece of ice as decribed above. The ice was produced at a relitively high ambient tunnel temperature $\left(-5^{\circ} \mathrm{C}\right)$, a moderate tunnel wind speed $\left(50 \mathrm{~m} \cdot \mathrm{s}^{-1}\right)$ and relatively low LWC $\left(0.3 \mathrm{~g} \cdot \mathrm{m}^{-3}\right)$. The resulting ice had a transparent aspect but was not completely glaze. The deposit temperature, measured with a thermocouple placed inside the metallic cylinder (on which the ice was accreted on) close to the surface, was slightly below the melting point of ice at $-1^{\circ} \mathrm{C}$. The freezing fraction (fraction of the liquid water that becomes ice on impingement) was estimated around 0.5.

Figure 3 shows the aspect of the grain at the interface ice/substrate. The polycrystalline structure of this type of ice was clearly visible. The average size of the grains 179 was $155 \mu \mathrm{m}$ in the plane of the section. The straight hor- 180 izontal lines and the fading of light in some part of the ${ }_{181}$ picture is an artefact effect due to the mosaic composi- 182 tion of the photos. The microstructure of the far side of ${ }_{183}$

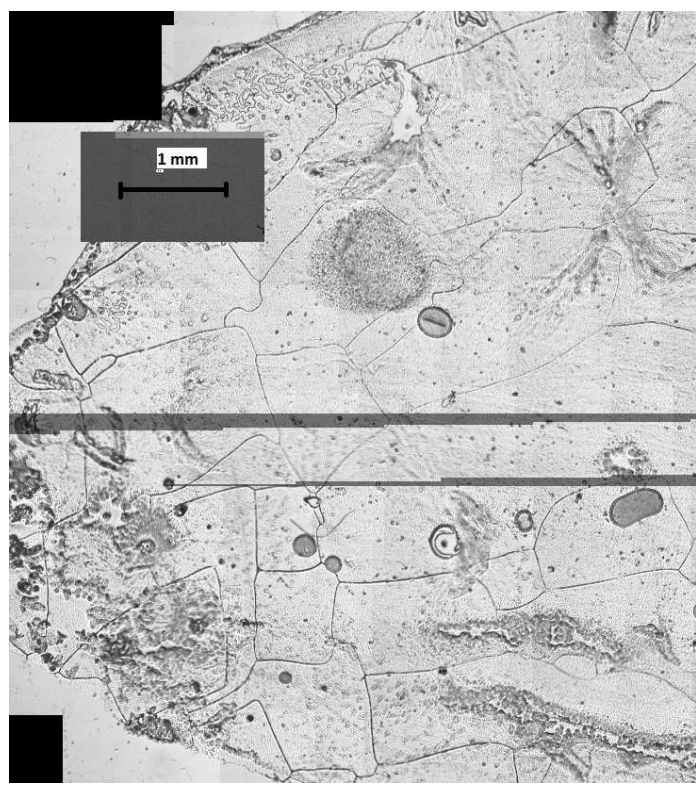

Figure 4: Microstructure of the front face of ice built on a mirror polished surface finish titanium alloy substrate $\left(T=-5^{\circ} \mathrm{C}, \mathrm{LWC}=0.3\right.$ g. $\mathrm{m}^{-3}, V=50 \mathrm{~m} \cdot \mathrm{s}^{-1}, M V D=20 \mu \mathrm{m}$ )

va Another feature that can be observed using the nail
varnish replica method is the presence and the size of air pockets within the ice. These air pockets can be of different kind and differ mainly by the way they are formed. One of the kind is due to air contained in 


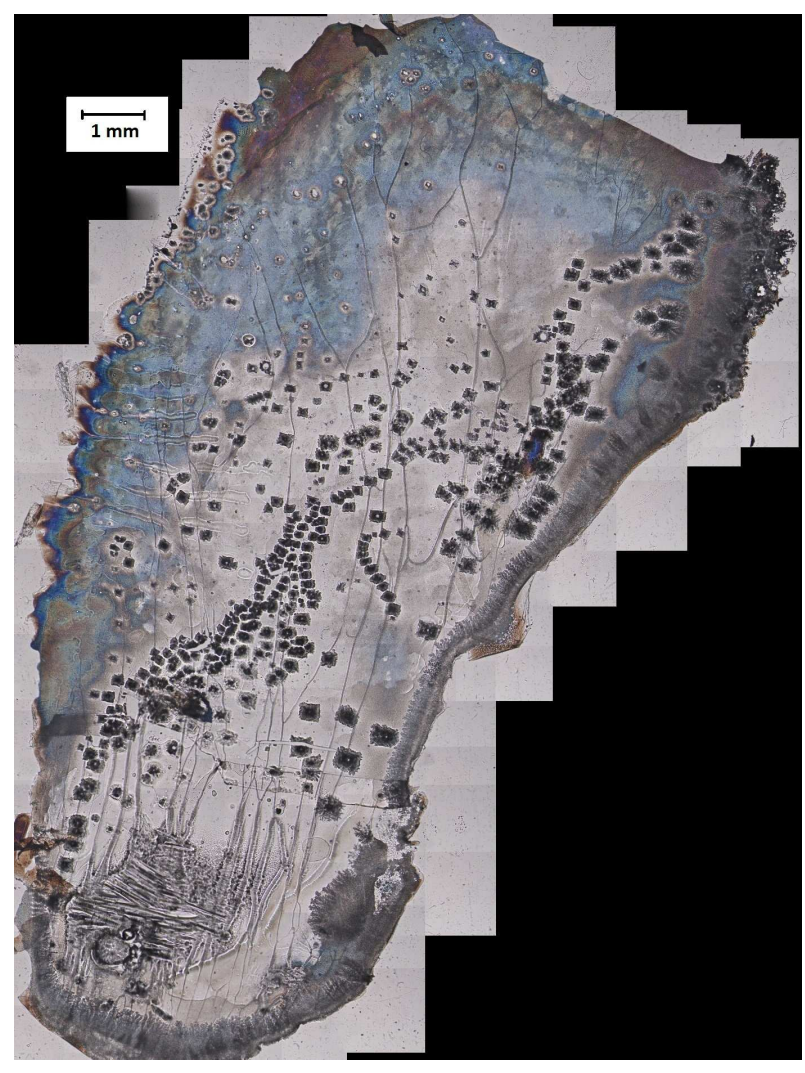

Figure 5: Microstructure of the longitudinal cut of ice built on a mirror polished surface finish titanium alloy substrate $\left(T=-5^{\circ} \mathrm{C}, L W C=0.3\right.$ g. $\mathrm{m}^{-3}, V=50 \mathrm{~m} \cdot \mathrm{s}^{-1}, M V D=20 \mu \mathrm{m}$ )

the water droplets which is dissolved during the ice accretion and remained trapped within the ice (Eskandarian and Farzaneh, 2005; Macklin, 1962). Another kind which happens mainly with rime ice at low LWC, is formed when the water droplets impinge the surface and dry patches are created between each droplet.

Tiny air bubbles can be seen on figure 6 as small black dots especially present along the grain boundaries. This picture represents the interface part of an ice sample grown on mirror polished aluminium alloy at a tunnel total temperature of $-20^{\circ} \mathrm{C}$, a speed of $50 \mathrm{~m} \cdot \mathrm{s}^{-1}$, a LWC of $0.3 \mathrm{~g} . \mathrm{m}^{-3}$ and a droplet mean volume diameter of 20 $\mu \mathrm{m}$. These icing conditions resulted in an ice sample which had a white appearance and a freezing fraction of 1. This means that each droplets coming into contact with the metalic surface would freeze competely when impinging the surface. This type of ice is commonly refered as rime ice. On this particular example, the average size of the grains was $52 \mu \mathrm{m}$ and the size of the air bubbles was around $10 \mu \mathrm{m}$.

Another type of bubbles, more accurately referred 210

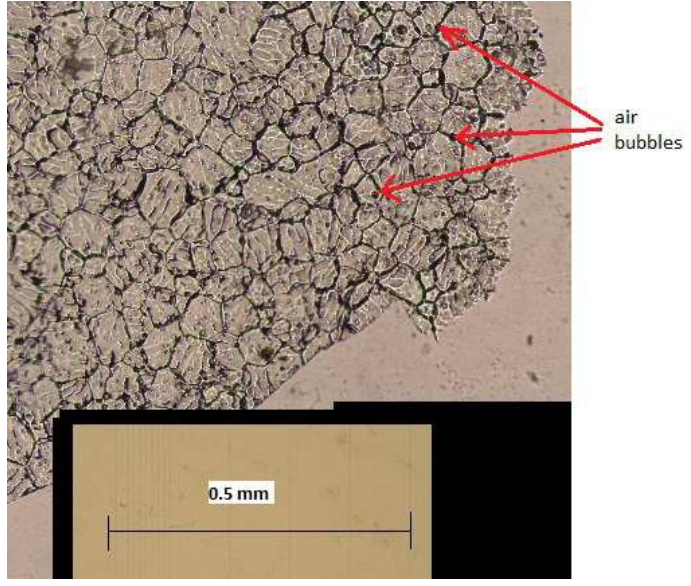

Figure 6: Microstructure of the interface of ice built on a mirror polished surface finish aluminium alloy substrate $\left(T=-20^{\circ} \mathrm{C}, L W C=0.3\right.$ g. $\mathrm{m}^{-3}, V=50 \mathrm{~m} . \mathrm{s}^{-1}, M V D=20 \mu \mathrm{m}$ )

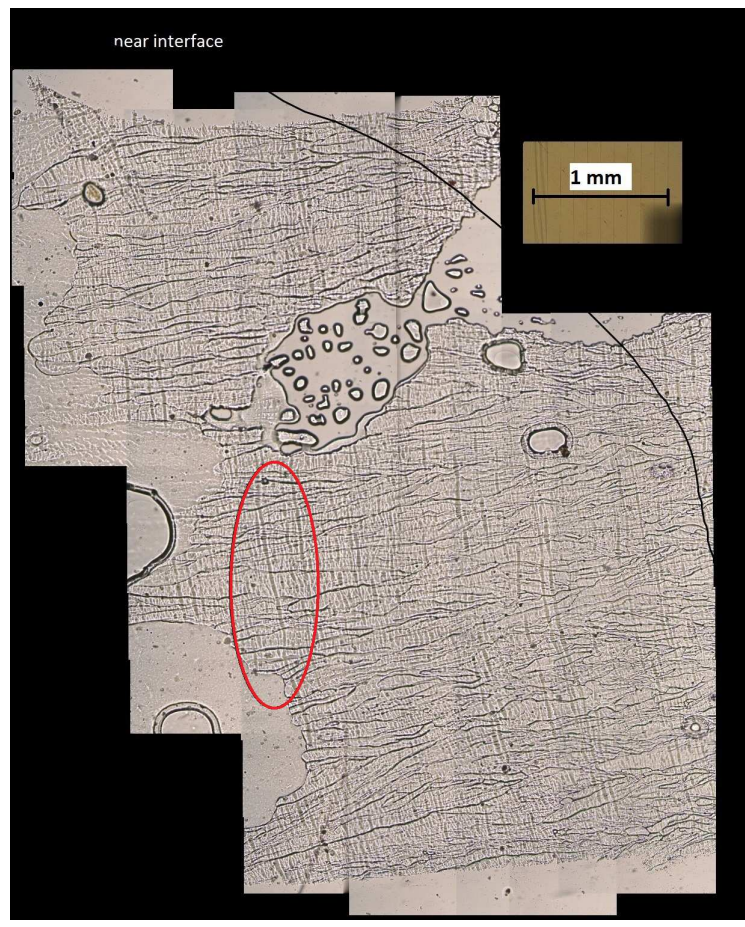

Figure 7: Microstructure of the longitudinal cut of ice built on a mirror polished surface finish titanium alloy substrate $\left(T=-15^{\circ} \mathrm{C}, L W C=0.3\right.$ g. $\mathrm{m}^{-3}, V=80 \mathrm{~m} \cdot \mathrm{s}^{-1}, M V D=20 \mu \mathrm{m}$ )

as porosity and described previously by Laforte et al. (1983), can be spotted on figure 7 (an example of them has been circled in black on the figure). This picture represents the microstructure of a longitudinal cut of a sample of ice grown on mirror polished titanium alloy at a temperature of $-15^{\circ} \mathrm{C}$. The grains here were columnar 
and relatively elongated in respect to their width (as- 242 pect ratio of around 20). A few of the black round dots 243 described before can be spotted but more interestingly, 244 some faint wavy vertical lines can be observed almost 245 perpendicular to the grain boundaries. These are made of tiny bubbles and represent variations in fine porosity. They also allow us to visualise the advancement of the growth of the ice front.

\subsection{Thermal etch pits}

The last feature described in this paper have been previously reported by Nasello et al. (1987) and Kriston et al. (2016). It was only observed when the size of the grains were resonably wide (above $100 \mu \mathrm{m}$ ).

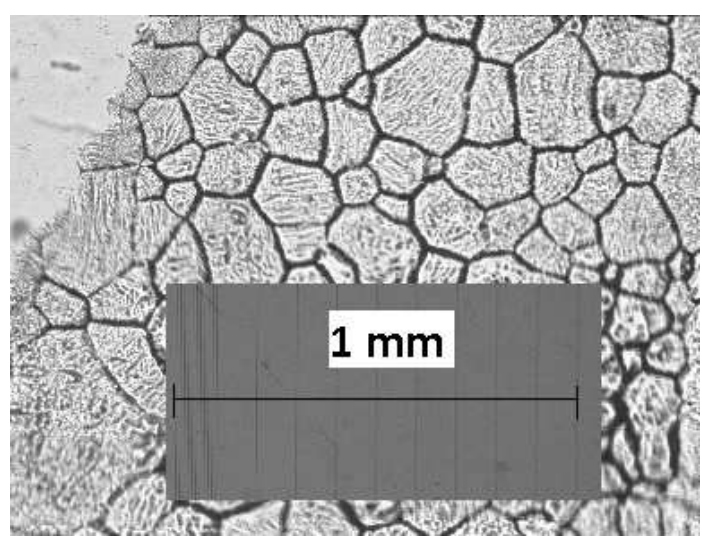

Figure 8: Microstructure of the interface of ice built on a mirror polished surface finish aluminium alloy substrate $\left(T=-10^{\circ} \mathrm{C}, \mathrm{LWC}=0.8\right.$ g. $\mathrm{m}^{-3}, V=50 \mathrm{~m} . \mathrm{s}^{-1}, M V D=20 \mu \mathrm{m}$ )

On figure 8, faded greyish non continuous small lines can be spotted inside most of the grains. These are thought to be arrays of thermal etch pits and can give some indication of the orientation of the crystal c-axis.

\section{Conclusion}

It has been demonstrated that nail varnish may be used to observe the microstructure of atmospheric ice. This method reduced considerably the preparation of the sample compared to more conventional method used previously to observe the microstructure of ice. Furthermore the ice replicas are not destroyed by time as would a thin slice of ice and can be observed as many times as needed.

These replicas allow the observation of many features as the size and shape of the grains, the presence of air inclusions, the growth lines of ice and the thermal etch pits that give an indication of the orientation of the caxis.

The technique offers a relatively simple way to observe ice microstructures so that it may be possible for more research on ice feature to be supported with reporting ice microstructural information.

\author{
246
}


2017-05-13

\title{
Observation of microstructures of atmospheric ice using a new replica technique
}

\author{
Pervier, Marie-Laure
}

Elsevier

Pervier MA, Pervier H, Hammond DW. (2017) Observation of microstructures of atmospheric ice using a new replica technique. Cold Regions Science and Technology, Volume 140, August 2017, pp. 54-57

http://dx.doi.org/10.1016/j.coldregions.2017.05.002

Downloaded from Cranfield Library Services E-Repository 